\title{
Elastic-Plastic Behaviour of Structural Members and Systems with Crack Damage (1st Report)
}

\author{
by Tetsuya Yao*, Member Torgeir Moan**
}

\begin{abstract}
Summary
Crack damages are sometimes detected in offshore structures for petroleum activities. In this paper, influences of cracks on the performance of structural members and systems are theoretically investigated. A computer program SYSTAN 2 based on the Idealized Structural Unit Method is developed for an elastic-plastic analysis of two-dimensional structural systems containing cracked members. The influence of cracks on the full plastic strength interaction relationships is considered.

Example calculations are carried out with SYSTAN2. First, the ultimate strength of a both ends simply supported tubular member with a circumferential through-thickness crack is evaluated under axial load. It has been found that compressive ultimate strength depends on the slenderness ratio of a member and the magnitude of initial deflection as well as the crack size. On the other hand, tensile ultimate strength depends only on the size of a crack.

A quasi-dynamical analysis is also performed for the transverse section of a semi-submersible drilling unit with assumed crack damages in the lower horizontal brace. The following observations have been made :

(1) The intact structure behaves elastically under the extreme cyclic wave loading.

(2) With a moderate crack damage in the brace, the cumulated plastic deformation at every wave cycle levels off after some wave cycles.

(3) With a more significant crack damage, the progressive plastic collapse of the transverse section takes place.

Further work is needed to account for the elastic-plastic crack tip behaviour and the crack growth effect.
\end{abstract}

\section{Introduction}

Offshore structures used in petroleum activities are usually subjected to various loads such as functional loads and environmental loads. In general, it may be said that no buckling or plastic collapse takes place at their legs or bracing members under these loads. The possible damage modes for such structural members are the initiation of fatigue cracks due to wave induced cyclic loads and/or the formation of local denting and overall bending damages due to minor supply boats collisions or falling objects from a deck.

These damages usually may not have a serious influence on the performance of the damaged members at the time of their formations. However, they may later affect the load carrying capac-

* Hiroshima University

** University of Trondheim/The Norwegian Institute of Technology ity of the damaged members under extreme sea conditions, which in some cases leads to the loss of integrity in the structural system as a whole.

The existing petroleum platforms can not be free from such kinds of damages, and some repairs may be necessary in some cases. However, it is very difficult and expensive to repair damaged structural members at sea. So, from the view point of safety as well as economy avoiding unnecessary and expensive repairs at sea, it is very important and urgent to be able to assess the influence of damages on the performance of damaged structural members and/or systems.

Local denting and overall bending damages reduce the load carrying capacity of damaged tubular members subjected to compressive axial force significantly. Theoretical and experimental investigations performed during last several years to clarify this matter are reviewed in Refs. $1 \sim 3$ ).

On the other hand, the initiation of a crack results in the reduced cross sectional area and 
stress concentrations. Fracture mechanics analysis is commonly applied to deal with such problems. Much research work has been performed up to now, of which basic object is to find out the conditions for stable and unstable crack growths. Here, however, only the effect of a crack as the reduction in the cross sectional area is considered assuming the necessary ductility of the material, and the crack growth is not considered. It should be noticed that a full plastic collapse at a cracked cross section without separation may also cause the loss of load carrying capacity of a cracked member subjected to tensile axial force and bending moment. At the same time, cracks may also affect the buckling strength of a member when compressive axial force is applied $^{4), 5)}$. In this connection, full contact between two surfaces of a crack is assumed when the crack is subjected to compressive stresses.

In this paper, the attention is focussed on the assessment of plastic and buckling strength of cracked members as well as the residual strength of structural systems containing cracked members. A Computer program SYSTAN 2 is developed to perform theoretical analysis based on the Idealized Structural Unit Method (6),7). In the formulation of the stiffness equation, the influence of initial deflection is introduced. The full plastic strength interaction relationships are derived considering the influence of a crack.

A series of example calculations is carried out. First, the influence of a crack on the ultimate strength (plastic and/or buckling strength) is examined for tubular members subjected to axial load. A quasi-dynamical system analysis is also performed on the transverse section of an existing semi-submersible drilling unit subjected to cyclic wave loading, and the influence of cracks on its behaviour is discussed.

It should be noticed that the influence of crack growth is not taken into account in the present paper.

\section{Method of theoretical analysis}

\subsection{Method of system analysis}

Main strength members in offshore structures are tubular and girder members. As an efficient and accurate method for analizing such structures, the Idealized Structural Unit Method (ISUM) has been proposed ${ }^{6) .71}$. In this paper, a computer program SYSTAN 2 is developed based on the ISUM to perform two-dimensional analysis of structural systems with crack damages. In SYSTAN 2, two types of elements are incorporated, which are tubular elements and girder elements. In the derivation of a stiffness relation, the influence of initial deflection is introduced ${ }^{3) .8)}$.
Here, it should be noticed that the beam-column theory is applied to derive the stiffness matrix of a structural member in the ISUM. According to the beam-column theory, a plane perpendicular to a neutral axis is assumed to remain as a plane during deformation. This assumption implies the fact that a crack has no influence on the elastic behaviour of a structural member. In the actual case, local plastification may take place around the crack tip due to stress concentration from the early stage of loading. To take this phenomenon into account exactly, threedimensional shell analysis is necessary. However, such an analysis requires large amount of time and efforts, and can not be incorporated in the system analysis. So, in this paper, the beamcolumn theory is applied in the framework of the ISUM to derive the elastic stiffness matrix of a structural member neglecting the influence

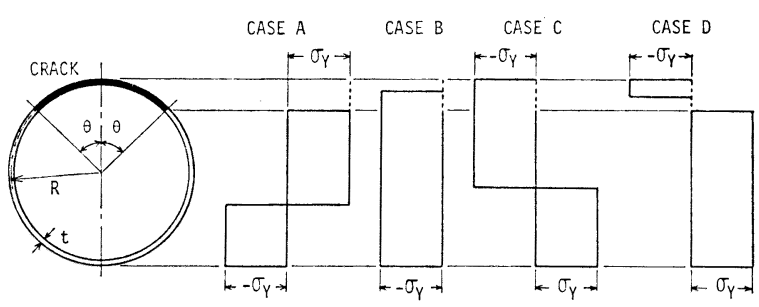

(a) Full plastic stress distributions

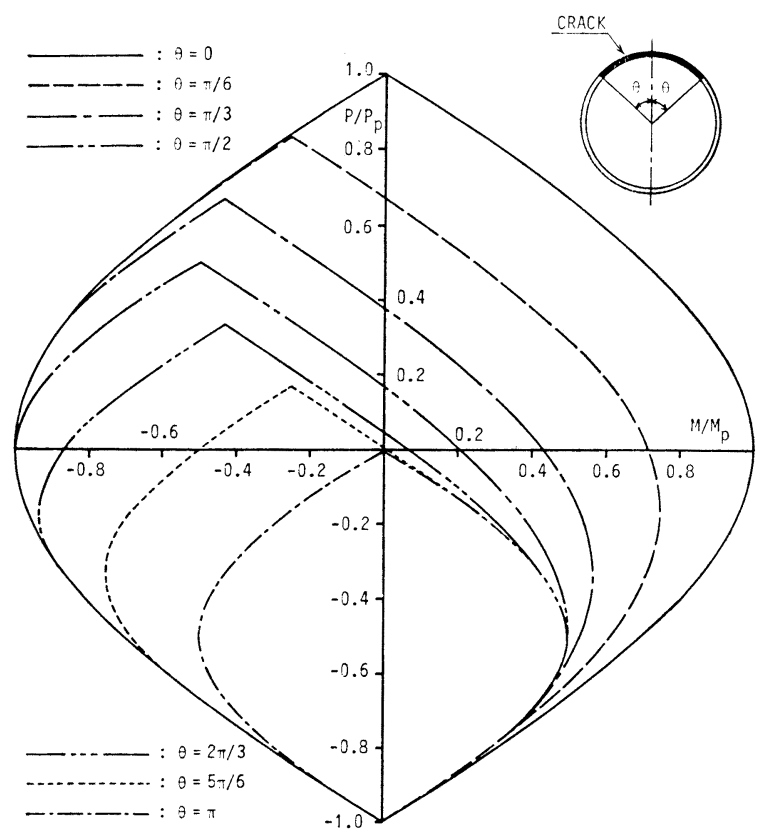

(b) Full plastic strength interaction curves

Fig. 1 Full plastic strength interaction relationships of tubular section with crack

of local plastification around the crack tip. The 
influence of a crack is taken into account when the full plastic condition is considered.

\subsection{Full plastic strength interaction rela- tionships}

In this section, full plastic strength interaction relationships are derived for a tubular cross section under general combinations of axial force and bending moment considering the influence of a crack. The material is assumed to be elasticperfectly plastic.

Here, a through-thickness crack is supposed to be located in the circumferential direction. A crack will initiate at the region where stresses are in tension. However, this region may also be subjected to compressive stresses after the crack initiation according to loading conditions. For such a situation, there may be two mechanics regarding the load carrying capacity at crack surfaces. One is that crack surfaces can carry neither compressive stresses nor tensile stresses. Another alternative is that they can carry compressive stresses but not tensile stresses. This may depend mainly on the magnitude of locally accumulated plastic deformation at a crack tip during cyclic loading. In this paper, however, crack surfaces are supposed to carry compressive stresses completely.

Then, there exist four possible full plastic stress distributions as illustrated in Fig. 1(a). In Cases $A$ and $C$, the whole crack is under tensile and compressive stresses, respectively, while a crack is partly under tensile and partly under compressive stresses in Cases B and D. For each stress distribution, full plastic strength interaction relationships between axial force and bending moment are given as :

Case A stress distribution

$$
\Gamma=m-\cos \left(\frac{\theta}{2}+\frac{\pi}{2} n\right)+\frac{1}{2} \sin \theta=0
$$

Case $B$ stress distribution

$$
\Gamma=m+\frac{1}{2} \sin \pi n=0
$$

Case $\mathrm{C}$ stress distribution

$$
\Gamma=-m-\cos \frac{\pi}{2} n=0
$$

Case D stress distribution

$$
\Gamma=-m-\frac{1}{2} \sin (\theta+\pi n)-\frac{1}{2} \sin \theta=0
$$

In the above expressions, $n=P / P_{P}$ and $m=M / M_{P}$ are the non-dimensionalized axial force and bending moment, and

$P_{P}=2 \pi \sigma_{Y} R t:$ full plastic axial force

$M_{P}=4 \sigma_{Y} R^{2} t:$ full plastic bending moment

Although the same kinds of interaction relationships are derived also for a girder cross section ${ }^{91}$, the results are not presented here.

The interaction relationships calculated by Eqs. (1) - (4) are plotted in Fig. 1(b). The crack angle $\theta$ is changed from 0 (no crack) to $\pi$ (complete separation).

\section{Example calculations and discussions}

\subsection{Ultimate strength of a cracked tubular member under axial load}

Using SYSTAN2, the ultimate strength of a cracked tubular member is first evaluated under axial compression and/or tension. The length, the mid-thickness diameter and the thickness of a member are taken as $35 \mathrm{~m}, 1,274 \mathrm{~mm}$ and $24 \mathrm{~mm}$, respectively, and the yield stress of the material as $240 \mathrm{~N} / \mathrm{mm}^{2}$. Both ends are supposed to be simply supported, and a crack is placed at the center of a span. In the analysis, initial deflection of a sinusoidal form is assumed as :

$$
w_{0}=a_{0} \sin \frac{\pi x}{L}
$$

where $a_{0}$ is taken as $\pm L / 1,000$ here.

The calculated results are plotted by $\bigcirc, \triangle$ and $\diamond$ in Figs. 2(a) and (b). In these figures, loaddeflection curves and relationships between axial force and bending moment are presented, respectively.

For this simple example, analytical solutions are also derived. That is, at a cracked section, the elastic load-deflection relationship is given as:

$$
\bar{w}=\frac{\pi \bar{a}_{0}}{1+\lambda^{2} n}
$$

and the elastic axial force-bending moment relationship as :

$$
m=-\frac{\pi \bar{a}_{0} n}{1+\lambda^{2} n}
$$

where $\bar{w}=w / D$ and $\bar{a}_{0}=a_{0} / D$ are non-dimensionalized total and initial deflections, and

$$
\begin{aligned}
& \lambda=\sqrt{P_{E} P_{P}}=\frac{L}{\pi r} \sqrt{\frac{\sigma_{Y}}{E}}: \text { slenderness ratio } \\
& r=\sqrt{\frac{I}{A}} \text { : radius of gyration }
\end{aligned}
$$

On the other hand, in the plastic range, relationships between axial force and bending moment are given by Eqs. (1) (4). The load-deflection relationships are obtained through $M=-P \cdot w$, and are expressed as follows.

Case A stress distribution

$$
\bar{w}=-\frac{1}{\pi n}\left[\cos \left(\frac{\theta}{2}+\frac{\pi}{2} n\right)-\frac{1}{2} \sin \theta\right]
$$

Case $\mathrm{B}$ stress distribution

$$
\bar{w}=\frac{1}{2 \pi n} \sin \pi n
$$




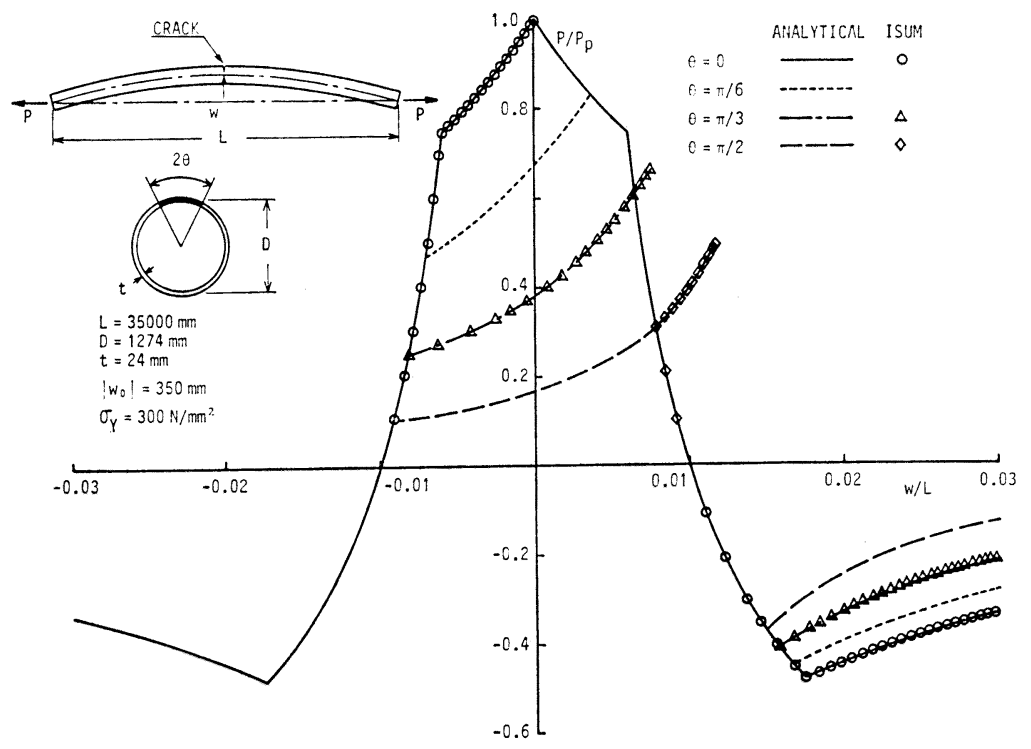

(a) Load-deflection curves

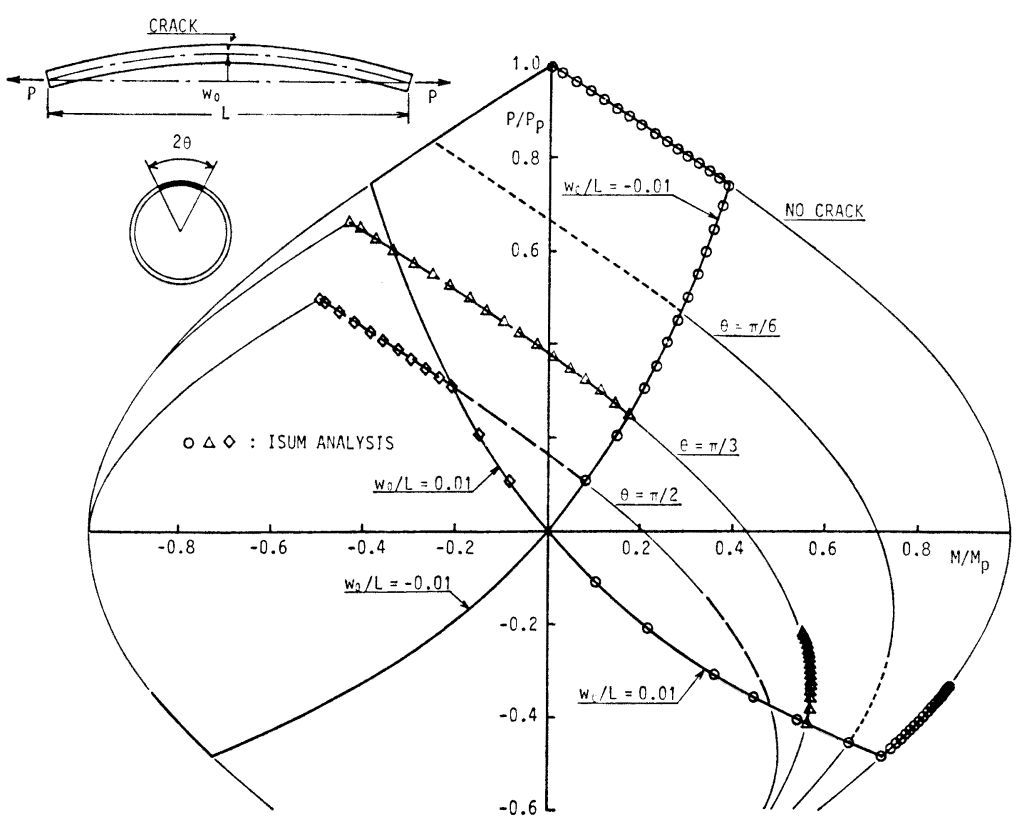

(b) Axial force-bending moment curves at cracked section

Fig. 2 Elastic-plastic behaviour of cracked tubular member under axial load

Case $\mathrm{C}$ stress distribution

$$
\bar{w}=\frac{1}{\pi n} \cos \frac{\pi}{2} n
$$

Case D stress distribution

$$
\bar{w}=\frac{1}{2 \pi n}[\sin (\theta+\pi n)+\sin \theta]
$$

The curves in Figs. $2(\mathrm{a})$ and (b) are the results calculated by the above equations. It is found that the incrementally calculated results by SYS-
TAN 2 are accurate enough.

Here, the intersecting point of the elastic and the plastic curves may give the ultimate strength when the axial force is in compression. In actual structural members, however, local plastification preceedes in a crack tip region due to stress concentration. As a result, larger deflection is produced than that calculated by Eq. (8), and the real compressive ultimate strength may become 


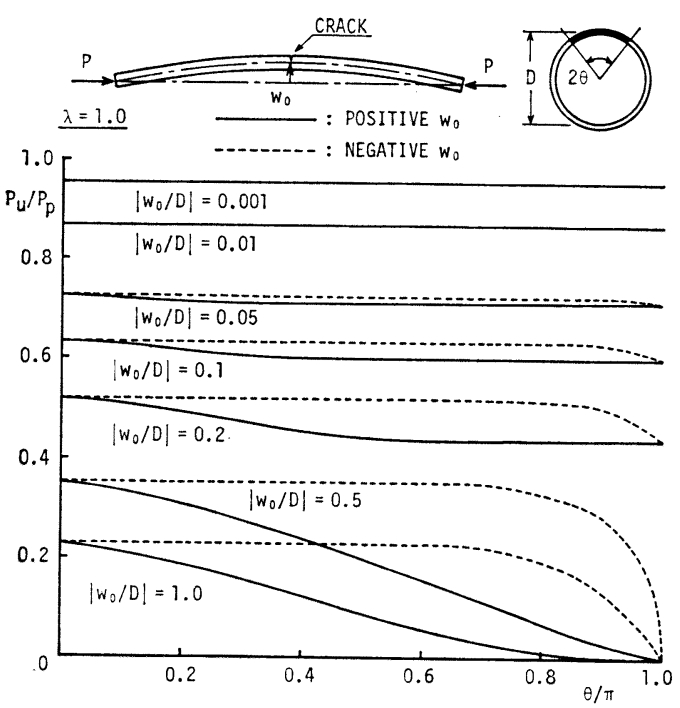

(a) Compressive ultimate strength

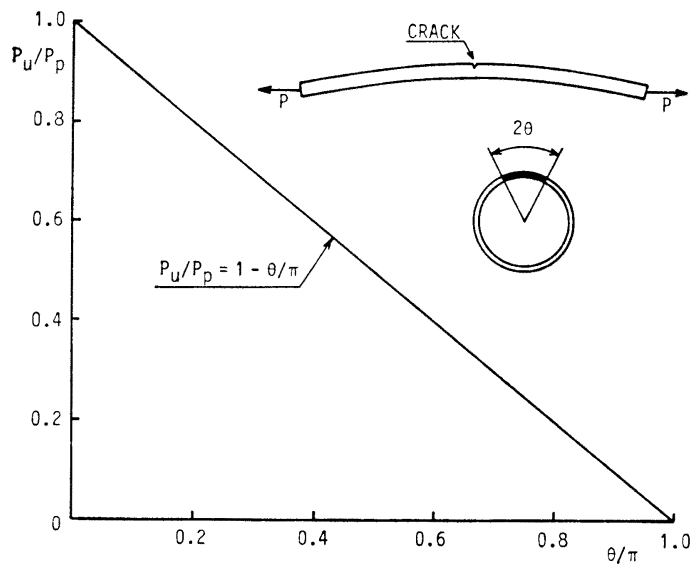

(b) Tensile ultimate strength

Fig. 3 Ultimate strength of cracked tubular member under axial load

lower than that evaluated here. To examine the accuracy of the evaluated ultimate strength, some experiments and more exact calculations are necessary. It should be noticed that the compressive ultimate strength evaluated here is an upper bound solution.

The compressive ultimate strength is evaluated as a function of the slenderness ratio, $\lambda$, and the magnitude of initial deflection. Here, the results for $\lambda=1.0$ are shown in Fig. 3 (a). When the magnitude of initial deflection is small, a non zero ultimate strength may be expected even when $\theta=\pi$ (completely separated at a cracked section). On the other hand, it falls down to zero when the initial deflection is large. The limiting magnitude of initial defection is evaluated from the condition that the elastic load-deflection curve has an intersecting point with the plastic one. It is given as :

$$
a_{0 \imath \imath \mathrm{m}}=\frac{D}{2}
$$

Contrary to this, the intersecting point of the elastic and the plastic curves does not give the ultimate strength when the axial force is in tension. In this case, the axial force increases also after the full plastic condition is satisfied, and the ultimate strength is given as:

$$
\frac{P_{u}}{P_{p}}=\frac{1-\theta}{\pi}
$$

Calculated results are shown in Fig.3(b). It should be noticed that tensile ultimate strength depends only on the size of a crack.

\subsection{Influence of cracks on the performance of a semi-submersible drilling unit}

3.2.1 Calculation model and loading conditions Example calculations are performed using SYSTAN 2 to examine the influence of cracks on the performance of an existing semi-submersible drilling unit schematically illustrated in Fig. 4. Here, a two-dimensional analysis is carried out on the transverse section $A B C D$ of this drilling unit. $A$ beam sea condition with a regular wave is supposed, and the half wave length $L_{w} / 2$ is taken as the breadth of the transverse section. The dimensions and the discretization into idealized structural units are shown in Fig. 5.

To evaluate wave induced cyclic loads, however, three-dimensional analysis is carried out applying the linear wave theory. Here, it should be noticed that the three-dimensionally calculated wave induced loads are in equilibrium with the inertia forces calculated at the same time. So, if only the transverse section $A B C D$ is separated from the whole system, the three-dimensionally calculated wave induced loads and inertia forces may not be in equilibrium with each other. In this paper, the inertia forces are calculated to balance the wave induced loads acting on the transverse section ABCD. That is, the increments of wave induced loads during the time interval $\Delta T$ are

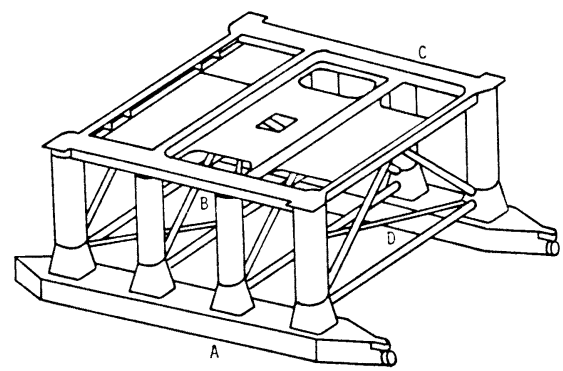

Fig. 4 Calculated model of semi-submersible drilling unit 


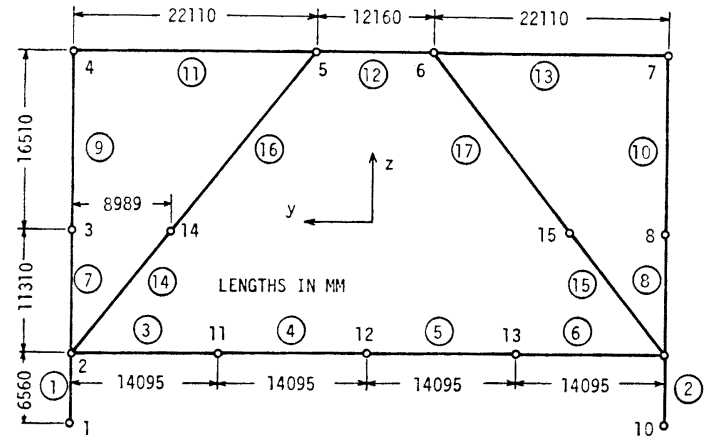

(a) Dimensions of transverse section

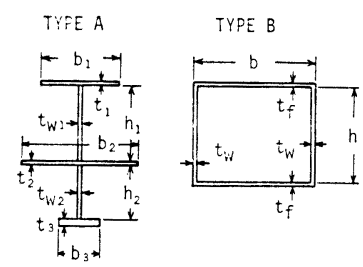

TYPE C

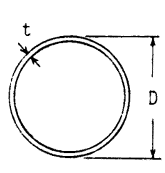

GIRDER ELEMENT - TYFE A

\begin{tabular}{ccccccccc}
\hline ELEMENT No. & $b_{1}$ & $t_{1}$ & $h_{1}$ & $t_{W_{1}}$ & $b_{2}$ & $t_{2}$ & $h_{2}$ & $t_{W 2}$ \\
\hline 11,13 & 1550 & 14.1 & 3040 & 10 & 3000 & 12 & 2000 & 11.2 \\
12 & 1550 & 13.5 & 3040 & 12 & 2500 & 12 & 2000 & 20 \\
\hline$b_{3}$ & $t_{3}$ & & & & & & & \\
\hline 400 & 20 & & & & & & & \\
750 & 25.26 \\
\hline
\end{tabular}

(b) Dimensions of cross sections of elements

Fig. 5 Idealized structural unit representation of transverse section
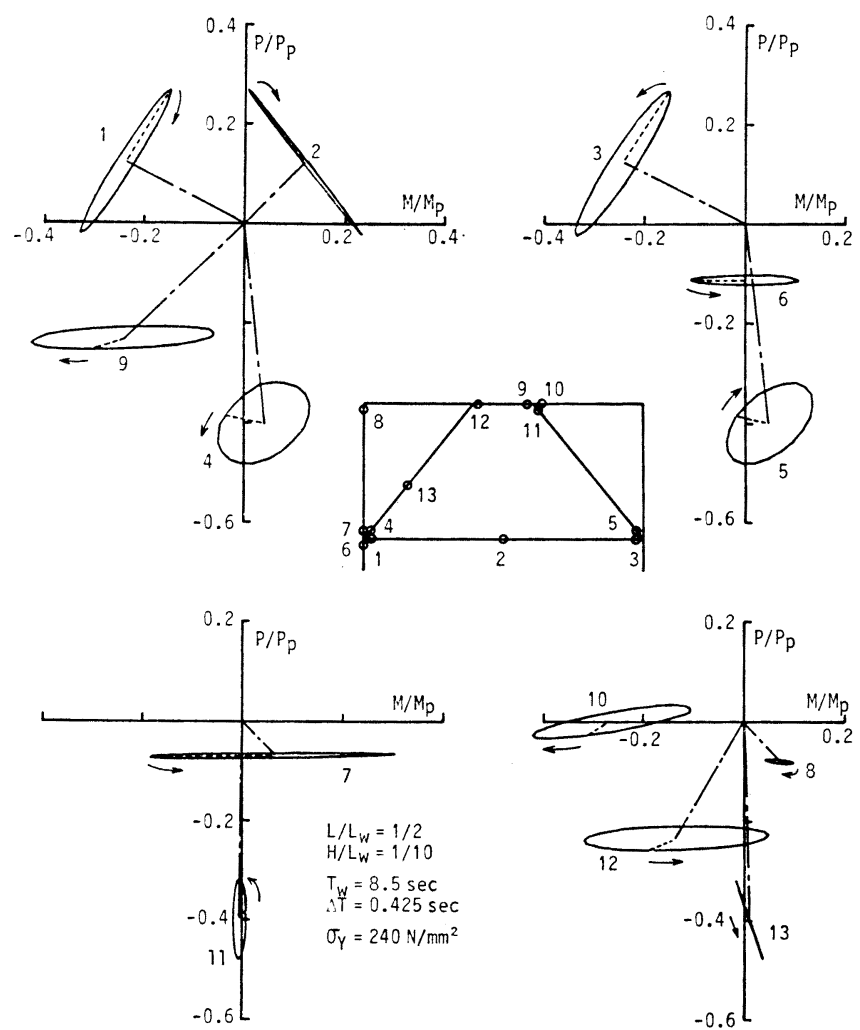

Fig. 6 Loci of internal forces at some points of intact transverse section under wave induced cyclic loads 
evaluated by subtracting the wave induced loads at the time $T$ from those at $T+\Delta T$. Then, accelerations are evaluated for these increments of wave induced loads at every time interval considering the equilibrium conditions of forces and moments acting on $A B C D$. The inertia forces necessary to balance the increments of wave induced loads at every time interval are calculated using the evaluated accelerations.

Considering that the loads are decomposed into the dead loads and the wave induced cyclic loads, the loading stage is divided into three parts, which are :

(1) Stage 1; apply dead loads incrementally

(2) Stage 2; apply wave induced loads at a specified initial time $T=T_{0}$ incrementally

(3) Stage 3 ; apply the increments of wave induced loads changing the time $T$ incrementally from $T=T_{0}$

Such an analysis may be denoted quasi-dynamical.

Here, to avoid the rigid body movement of a system and hence the ill-conditioned equilibrium equations, weak springs of $500 \mathrm{~N} / \mathrm{mm}$ are placed at the nodal points $1,4,7$ and 10 in $y$ - and $z$ directions, respectively. It has been confirmed that this magnitude of spring stiffness does not affect the behaviour of the transverse section and at the same time perform their functions.

In the following analysis, the wave height is taken as $H / L_{w}=0.1$, and the time interval as $\Delta T=T_{w} / 20$, where the wave period $T_{w}$ is 8.5 sec. . The yield stress of the material is assumed to be $240 \mathrm{~N} / \mathrm{mm}^{2}$.

\subsubsection{Behaviour of a transverse section free} from cracks

First, analysis is performed on a transverse sec- tion free from cracks. The relationships between the axial force and the bending moment at some nodal points are plotted in Fig. 6 . The chain lines, dashed lines and solid lines in the figure represent the paths of internal forces during stage 1,2 and 3 loadings, respectively. It is observed that the loci of internal forces at stage 3 loading represented by solid lines become closed curves of which one cycle corresponds to that of the wave.

Although the assumed loading condition may be the most severe one among existing loading conditions, no plastification takes place. It may be said that this semi-submersible drilling unit has enough transverse strength regarding to buckling and plastification even if the material of such low yield stress is employed.

According to the calculated results, the shear force in element 12 is rather large, and it will not be appropriate to neglect the influence of shear force on the full plastic strength as to this element. Therefore, the yield stress is reduced to $70 \%$ of the material regarding to element 12 in the following analysis. The internal forces at points 9 and 11 in Fig. 6 are non-dimensionalized by this reduced yield stress.

Here, an alternative analysis is carried out on an intact system. In this analysis, stage 3 loading is not performed, although the wave induced loads at $T=T_{0}=0$ are monotonically increased in stage 2 loading until a full plastic mechanism is formed as a whole. The wave height $H / L_{w}$ which is neces. sary to cause the plastic collapse of a system is $0.53^{9)}$. This wave height is unrealistic as an actual one, and it may be again concluded that this system has enough strength if it is free from any kind of damage.

3.2.3 Behaviour of a transverse section with

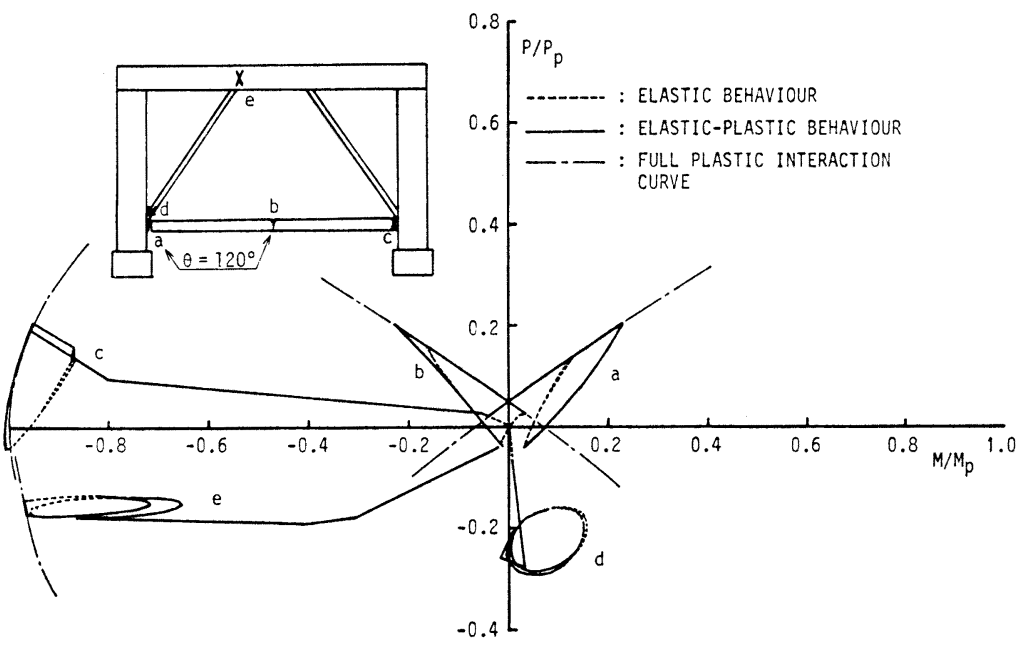

Fig. 7 Loci of internal forces under wave induced cyclic loads (Case I) 


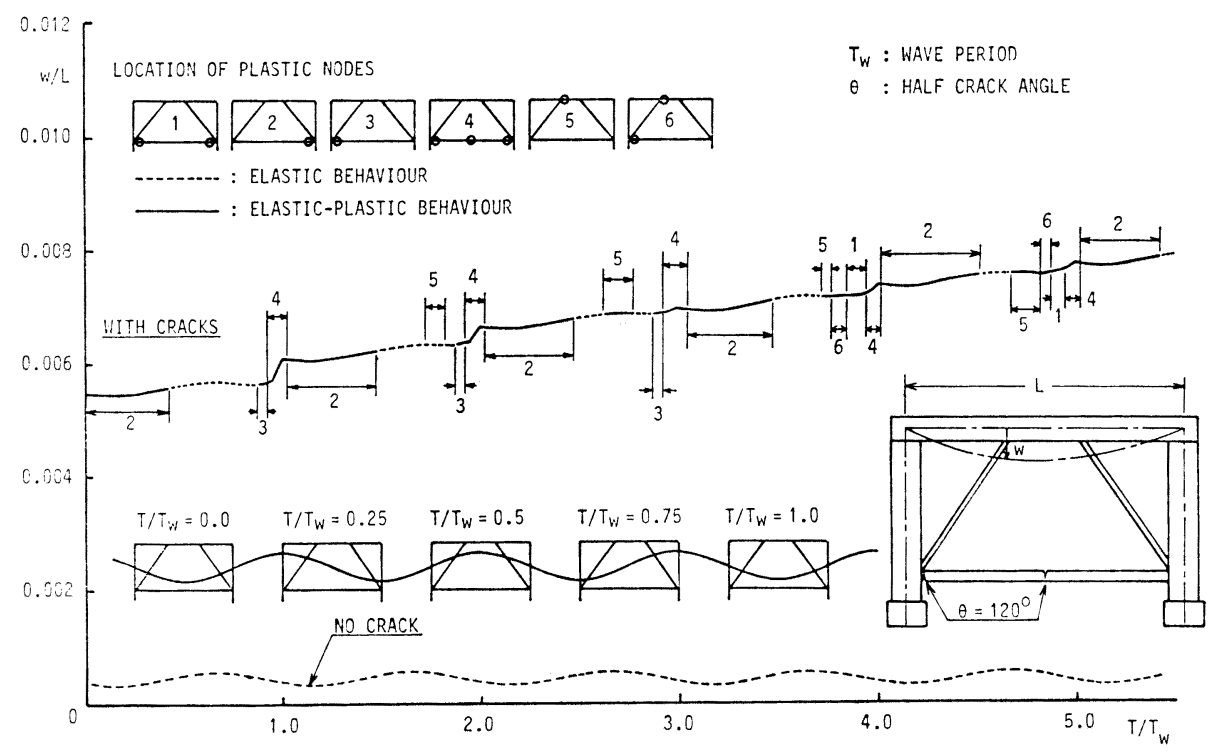

Fig. 8 Deflection of deck girder under wave induced cyclic loads (Case I)

cracked members

The high cycle stress variations in the tension range of an axial force in the lower horizontal brace suggest that fatigue cracks may mostly initiate there. Possible locations of the crack initiation along this bracing member are its both ends where it is connected to the column as well as its center where other bracing members are connected. In the following, two case are analyzed. They are :

Case I ; cracks of $\theta=2 \pi / 3$ at the upper side of a midspan and the lower side of one end

Case II ; a crack of $\theta=2 \pi / 3$ at the upper side of a midspan and cracks of $\theta=19 \pi / 90$ at the lower side of both ends

The assumed numbers and sizes of cracks may be a little unrealistic for the most of the actual cases. However, these excessive numbers and sizes are chosen to demonstrate typical features of cracks' influence on the performance of structural systems.

\section{Case I}

In the analysis, the initial time for stage 2 loading is defined as $T_{0} / T_{w}=0.0$. The calculated results are shown in Figs. 7 and 8 , where the loci of internal forces and the deflection of a deck girder are illustrated, respectively.

In this case, the plastifications occur at cracked sections during stage 1 loading, which results in large deviations of the loci of internal forces at all the points, especially at point c. As the result, point $\mathrm{c}$ becomes plastic at the end of stage 2 loading, and a plastic mechanism is formed at the horizontal brace. Due to this, the locus of internal forces at point e moves toward the full plastic strength interaction curve, but this point still remains elastic during stage 2 loading. At the beginning of stage 3 loading, unloading occurs at points $\mathrm{a}$ and $\mathrm{b}$. After $T / T_{w}=0.4$, point $\mathrm{c}$ is also unloaded, and the system behaves elastically until $T / T_{w}=0.85$. Then at $T / T_{w}=0.95$, a plastic mechanism is again formed at the horizontal brace, and there exists a rather large increase in the deflection of a deck girder.

Due to the plastification at point $\mathrm{c}$ until $T / T_{w}=$ 1. 45, the locus of internal forces at point $\mathrm{e}$ has moved outward, and a plastic node is formed at this point when $T / T_{w}=1.75$. At this time, however, a plastic mechanism is no more formed at the horizontal brace, and a plastic collapse of the system does not occur. At $T / T_{w}=1.95$, a plastic mechanism is again formed at the horizontal brace, but point $\mathrm{e}$ is unloaded this time.

Comparing the results in Figs. 6 and 7, shapes of the loci of internal forces at the yielded nodal points are different from those elastically calculated. They may depend on the shapes of full plastic strength interaction curves. At the same time, their locations are also different from the elastic ones. This is the result of redistributions of internal forces due to plastifications at some nodal points.

After $T / T_{w}=2.0$, however, further deviation of the loci of internal forces can hardly be observed, and almost the same behaviour is repeated at every wave cycle. It is seen that the increment of deflection decreases at every wave cycle, and the resulting accumulated deflection levels off after a number of wave cycles at least under the regular wave condition. 


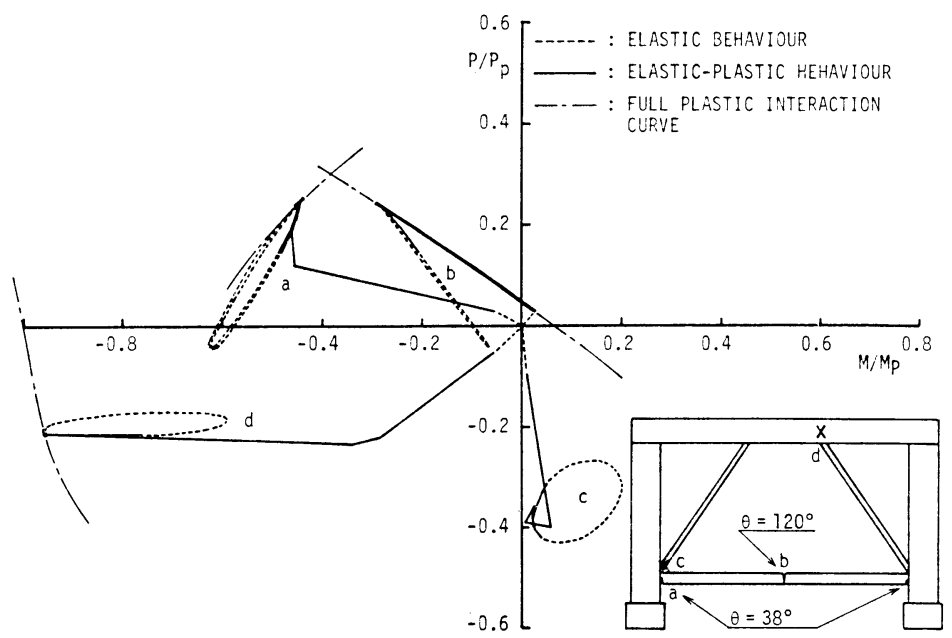

(a) $T_{0} / T_{w}=0.0$

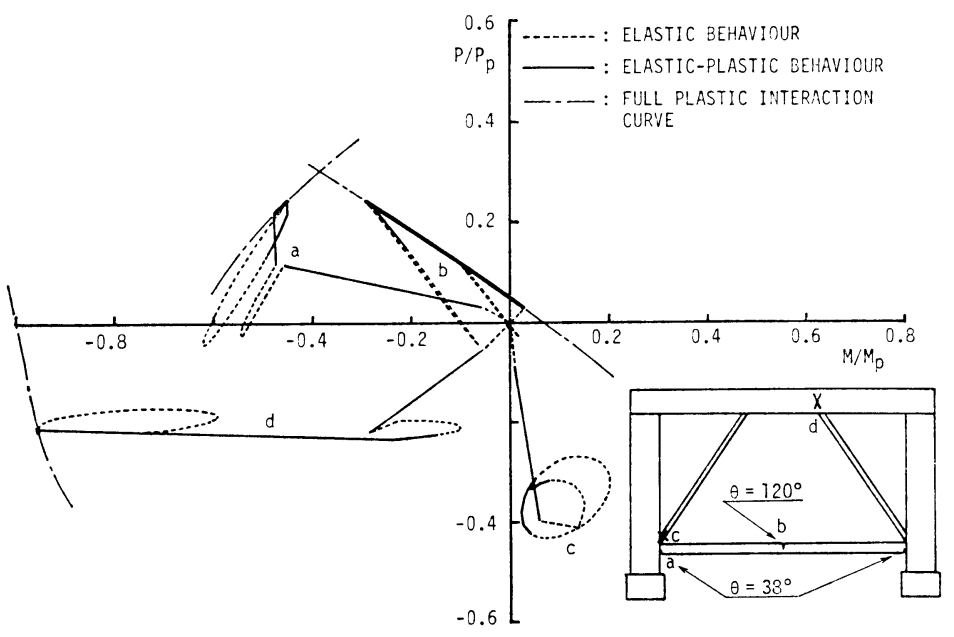

(b) $T_{0} / T_{w}=0.5$

Fig. 9 Loci of internal forces under wave induced cyclic loads (Case II)

In this case, plastic collapse of the system does not seem to take place.

Case II

For the analysis here, the initial time is chosen as $T_{0} / T_{w}=0.0$ and 0.5 . The calculated loci of internal forces are plotted in Figs. 9 (a) and (b), and the deflection of the deck girder in Fig. 10.

In both cases, the initial plastification takes place at the midspan cracked section, b, during stage 1 loading. When $T_{0} / T_{w}=0.5$, however, point $\mathrm{b}$ is unloaded at the beginning of stage 2 loading, and a system becomes to behave elastically. It is when $T / T_{w}=0.8$ in stage 3 loading that a plastic node is again formed at the center of the horizontal brace. Soon after this, both ends of the horizontal brace become plastic, and a plastic mechanism is formed in this member. Then, a plastic node is formed also at point $\mathrm{d}$ of a deck girder, and the transverse section becomes a plastic mechanism. Consequently, a rather large increase in the deflection of a deck girder is observed. However, a complete plastic instability does not occur owing to the elastic springs placed at the four corners of a system in two perpendicular directions. This may be in a sense realistic because this transverse section is supported by the longitudinal members. Some other plastic nodes may have to be formed in the other transverse and/or longitudinal sectiors so that a plastic instability of the whole system takes place.

Almost the same behaviour is repeated in the following wave cycles, and the deflection increases every time when a plastic mechanism is formed as a whole. Such a collapse may be denoted as 


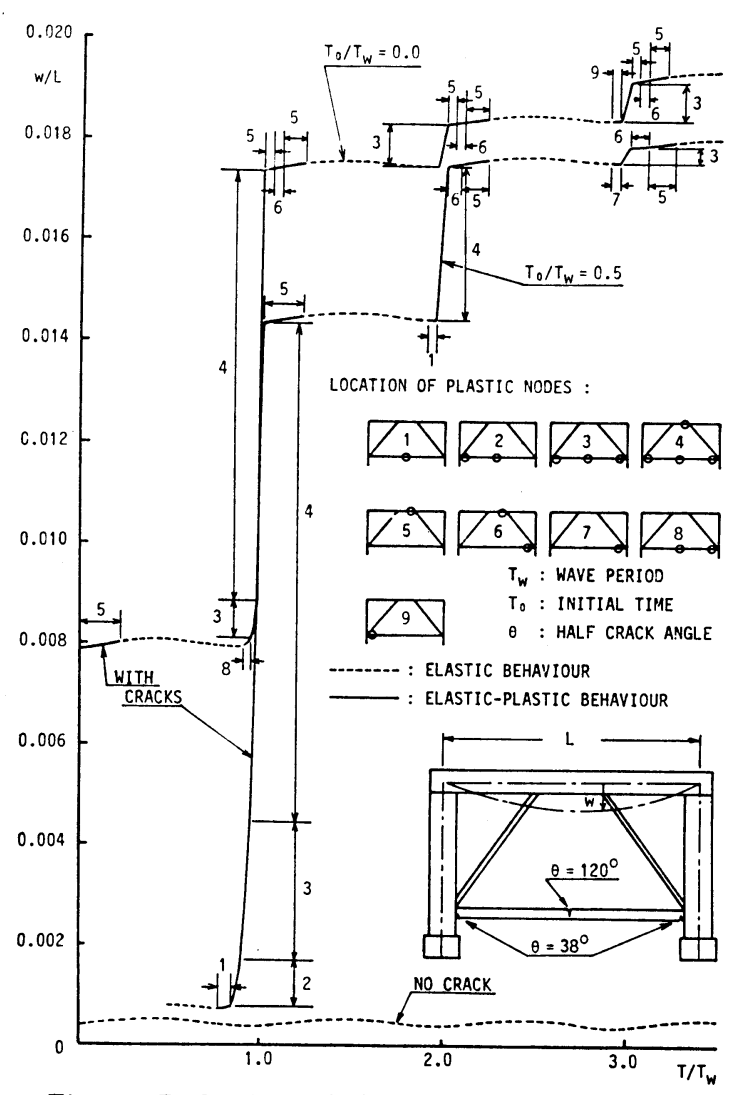

Fig. 10 Deflection of deck girder under wave induced cyclic loads (Case II)

a progressive plastic collapse. However, the magnitude of the increase in deflection becomes smaller and smaller at every wave cycle, and a system becomes plastically relaxed. This may be realistic when no more plastic node is formed at structural members in the longitudinal and/or other transverse sections.

On the other hand, when $T_{0} / T_{w}=0.0$, the first plastification at both ends of the horizontal brace occurs during stage 2 loading. Soon after this, point $\mathrm{d}$ also becomes plastic and a plastic mechanism of a whole system is attained in stage 2 loading. At the beginning of stage 3 loading, a plastic node remains only at point d. After $T / T_{w}=0.2$, this point is also unloaded, and the system behaves elastically until $T / T_{w}=0.9$. At the end of the first wave cycle, a full plastic mechanism of the system is again formed, and a large increase is observed in the deflection.

The difference in the initial time $T_{0}$ makes the loci of internal forces different before $T / T_{w}=$ 1.0 as represented in Figs. 9 (a) and (b). After $T / T_{w}=1.0$, however, the loci of internal forces in the two cases are almost the same in shape and location, and the behaviour of the system is also the same in both cases except the absolute value of deflection. Based on the above results and others not presented here ${ }^{9)}$, it may be concluded that the initial time $T_{0}$ for stage 2 loading affects the calculated behaviour after the second wave cycle, only to a small extent.

\section{2.4 Future works}

Besides on the analyses performed up to now, plastic collapse of the structural system with cracked members seems to take place in some cases. This may depend on the magnitude of plastic deformations at cracked cross sections accumulated at every wave cycle as well as the numbers and the sizes of cracks under the regular wave condition assumed here.

In a real case, however, the irregular wave condition has to be considered. It should be noticed that the irregularity of wave causes the differences in the formations of plastic nodes and their unloadings comparing to those under a regular wave condition.

In the analysis performed here, it is supposed that cracks have initiated due to high-cycle fatigue. Therefore, even if a plastic collapse of the system does not occur for a specified size of cracks, cracks may continue to grow under cyclic wave loads in the actual case. Consequently, a plastic collapse of the system may take place or ductile and/or brittle fracture of a cracked member may occur sooner or later after the crack has grown up to a certain size. To simulate such an actual behaviour of structural members and systems with crack damage, conditions for the crack growth in ductile and brittle manners have to be incorporated in the analysis. This remains as a very important and urgent future work.

In this paper, cracks are assumed at the locations where structural members are connected with each other. Crack initiation may alternatively take place at a dented part where large plastic deformation is produced during the denting damage. Regarding the initiation of a crack at a dent, experimental observations are necessary to develope relevant criteria. If the condition for this crack initiation has become clear, the same procedure may be applicable for the analysis of structural systems with dented members combining the proposed method with that for dented members ${ }^{3), 8)}$. This also remains as a future task.

\section{Conclusions}

In this paper, the influence of cracks on the elastic-plastic behaviour of structural members and systems is theoretically investigated. A computer program SYSTAN 2 is developed based on the Idealized Structural Unit Method. The influence of a crack is introduced in the full plastic strength 
interaction relationships.

Some example calculations are performed with SYSTAN 2 to examine the influence of cracks on the performance of structural members and systems. First, the ultimate strength of a cracked tubular member under axial load is evaluated under the assumption that compressive stresses are transfered between the crack surfaces, and that the material is perfectly ductile. It has been found that :

(1) Compressive ultimate strength depends both on the slenderness ratio of a member and the magnitude of initial deflection as well as the crack size.

(2) Tensile ultimate strength depends only on the size of a crack.

A quasi-dynamical analysis is also carried out on the transverse section of an existing semi-submersible drilling unit with assumed crack damages in its lower horizontal brace. The following informations have been obtained.

(1) If no crack exists, the transverse section behaves elastically under the extreme cyclic wave loading.

(2) With a moderate crack damage in the brace, the cumulated plastic deformation at every wave cycle levels off after some wave cycles.

(3) With a more significant crack damage, the progressive plastic collapse of the transverse section takes place.

No experiment is carried out to check the calculated results. It will remain as a future work. It also remains as a future work to extend SYSTAN 2 for three-dimensional analysis. Another important and urgent work is to incorporate the crack growth conditions in the analysis.

\section{Acknowledgements}

The authors wish to thank Dr. Gunnar Hessen for stimulating and helpful discussions. The financial support by the Royal Norwegian Council for Scientific and Industrial Research is also gratefully acknowledged.

\section{References}

1) Ueda, Y. and Rashed, S. M. H.: "Behaviour of Damaged Tubular Structural Members", OMAE (1985), pp. 528 536.

2) Taby, J. and Moan, T.: "Collapse and Residual Strength of Damaged Tubular Members", BOSS (1985), pp. 395 408.

3) Yao, T., Taby, J. and Moan, T.: "Ultimate Strength and Post Ultimate Strength Behaviour of Damaged Tubular Members in Offshore Structures", OMAE (1986), pp.301 308.

4) Lereim, J.: "Influence of Cracks on Structural Design Criteria", Int. Conf. on Application of Frac. Mech. to Materials and Structures, Freiburg, Germany (1983).

5) Lereim, J.: "Significance of Fracture Mechanics on Design of Offshore Structures", Int. Conf. on Fracture Prevention in Energy and Transport Systems, Rio de Janeiro, Brazil (1983).

6) Rashed, S. M. H.: "Behaviour to Ultimate Strength of Tubular Offshore Structures by the Idealized Structural Unit Method", Report SK/R 51, Division of Marine Structure, Norwegian Institute of Technology (1980).

7) Ueda, Y., Rashed, S. M. H. and Nakacho, K.: "New Efficient and Accurate Method of Nonlinear Analysis of Offshore Tubular Frame (the Idealized Structural Unit Method)", OMAE (1984), pp. $260 \sim 268$.

8) Yao, T., Fujikubo, M., Bai, Y. and Nakagawa, S.: "Load Carrying Capacity of Damaged Tubular Members", Trans. of The WestJapan Society of Naval Arch., Vol. 73 (1987), to be published (in Japanese).

9) Yao, T.: "Elastic-Plastic Analysis of Structural System Containing Cracked Members", Report of Division of Marine Structure, Norwegian Institute of Technology (1987), to be published. 